\title{
Raman fiber laser based on a 7-core fiber with fs-inscribed regular and random structures
}

\author{
A.V. Dostovalov ${ }^{1}$, M.I. Skvortsov ${ }^{1}$, A.A. Wolf ${ }^{1}$, V.I. Labuntsov ${ }^{1,2}$, O.N. Egorova $^{3}$, S.L. Semjonov ${ }^{4}$, \\ S.Wabnitz ${ }^{2,5}$, S.A. Babin ${ }^{1,2}$ \\ 1. Institute of Automation and Electrometry of the SB RAS, Novosibirsk, Russia \\ 2. Novosibirsk State University, Novosibirsk, Russia \\ 3. Prokhorov General Physics Institute, Russian Academy of Sciences, Moscow, Russia; \\ 4. Dianov Fiber Optics Research Center, Prokhorov General Physics Institute, Russian Academy of Sciences, Moscow, Russia \\ 5. Sapienza University of Rome, Rome, Italy
}

The significant progress in the development of multi-core fibers (MCFs) and the technology of refractive index change by fs- laser radiation [1] in the selective cores of MCF open up possibilities for the creation of new configurations of fiber lasers based on both active MCFs with inscribed fiber Bragg gratings [2] and passive MCFs in Raman lasers with random distributed feedback [3] for high-power laser applications.

In this work, we present the results of Raman fiber laser based on a 7-core fiber with coupled cores operating at $1090 \mathrm{~nm}$ pumped into central core by $\mathrm{Yb}$-doped fiber laser at $1050 \mathrm{~nm}$. The laser resonator is formed by highly reflective FBGs (>90\%) inscribed in the side cores of MCF used as the rear reflecting mirror and an array of highly reflective FBGs with a random displacement $(5-10 \mathrm{~mm})$ between them in the longitudinal direction with the most of power out-coupled trough the FBG-free central core. We also investigated a laser configuration with an additional fs-inscribed random structure of the refractive index in the central core of MCF provided a reflectance significantly higher $(>50 \mathrm{~dB})$ than the Rayleigh backscattering level.

The generation of single peak of $20 \mathrm{pm}$ linewidth (corresponding to OSA resolution) near the threshold was observed in both cases. At output power of 0.4 -1.5 W (from one fiber end), the laser linewidth (FWHM) is reduced by $\sim 2$ times in the case of laser cavity with random distributed feedback (RDFB) structure in central core of MCF (fig 1a, b). At Stokes output power of $2.25 \mathrm{~W}$, the difference between the linewidth became less about $50 \mathrm{pm}$, the FWHMs corresponded to $250 \mathrm{pm}$ and $300 \mathrm{pm}$, respectively (fig. 1c).
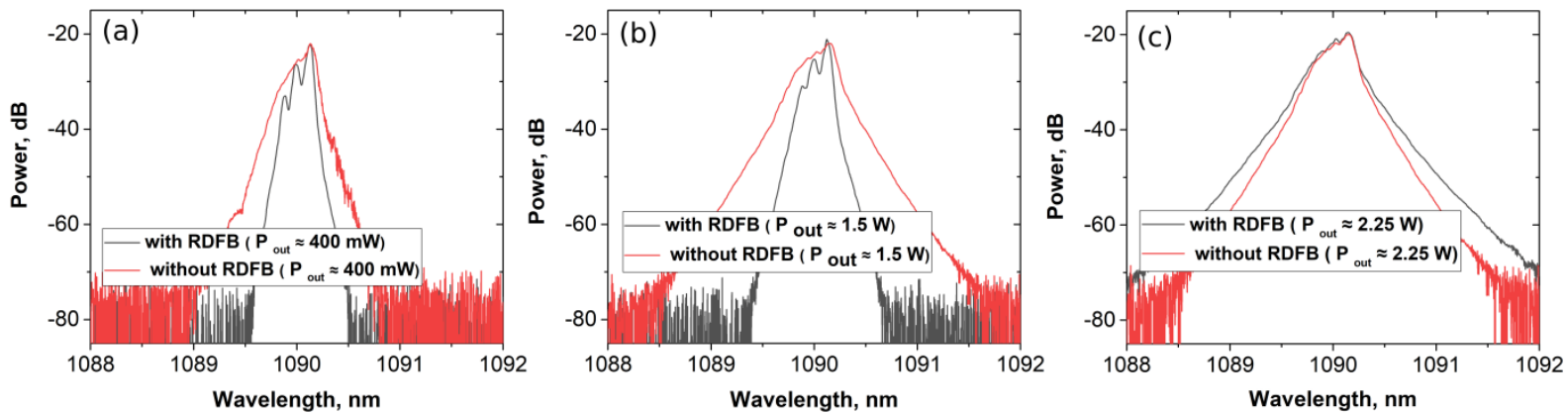

Fig. 1 The spectrum of Raman fiber laser with (black line) and without (red line) fs-inscribed random structure of the refractive index at different output power: a) $400 \mathrm{~mW}$, b) $1.5 \mathrm{~W}$, c) $2.25 \mathrm{~W}$.

Thus, we observe sufficient narrowing of the linewidth for MCF based Raman laser as compared to the random Raman lasers based on single- and twin- core fibers [4] that may be explained both by a decrease in the influence of nonlinear effects due to an increase of the effective mode field area in 7-core fiber and by the effect of spectral filtering of a fiber interferometer formed in a mode-coupled MCF by the FBG array with random axial displacements.

The work was supported by by the Russian Foundation for Basic Research (20-32-70132, 18-52-7822).

\section{References}

[1] A. Wolf, A. Dostovalov, K. Bronnikov, and S. Babin, "Arrays of fiber Bragg gratings selectively inscribed in different cores of 7-core spun optical fiber by IR femtosecond laser pulses," Opt. Express 27, 13978 (2019).

[2] Y. Alon, A. Halstuch, R. Sidharthan, S. Yoo, and A. Ishaaya, "Femtosecond Bragg grating inscription in an Yb-doped large-mode-area multicore fiber for high-power laser applications," Opt. Lett. 45, 4563-4566 (2020)

[3] M. I. Skvortsov, S. R. Abdullina, A. A. Wolf, A. V Dostovalov, A. A. Vlasov, I. A. Lobach, S. Wabnitz, and S. A. Babin, "Random Raman fiber laser based on a twin-core fiber with FBGs inscribed by femtosecond radiation," Opt. Lett. 44, 295 (2019).

[4] A. E. Budarnykh, I. A. Lobach, E. A. Zlobina, V. V. Velmiskin, S. I. Kablukov, S. L. Semjonov, and S. A. Babin, "Raman fiber laser with random distributed feedback based on a twin-core fiber," Opt. Lett. 43, 567 (2018). 